\title{
COMUNICACIÓN
}

\section{Cryptosporidium parvum en animales domésticos y en monos de un zoológico}

\author{
LUCILA VENTURINI*, D. BACIGALUPE*, W. BASSO, J. M. UNZAGA*, \\ M.C. VENTURINI* y G. MORÉ*
}

\section{Cryptosporidium parvum IN DOMESTIC ANIMAIS AND IN MONKEYS FROM A ZOO}

The aim of the present study was to detect Cryptosporidium sp infection in domestic animals and in monkeys from a zoo, in Buenos Aires Province, Argentina. Three hundred and seventy five fecal samples from different animal species were processed by the Ritchie modified sedimentation method (formalin-ether) to concentrate the oocysts. The sediment was stained by the Ziehl Neelsen modified technique. Cryptosporidium sp was detected in 7 of 175 dogs, in 2 of 17 cats, in 4 of 22 sheep, in 21 of 31 goats, in 29 of 109 calves, in 2 of 2 horses and in 2 of 5 Guinea pigs. Fourteen feces samples from monkeys were examined, among them, oocysts were detected in the sample from 1 adult female black howler monkey (Alouatta caraya), in the sample from 1 male adult black spider monkey (Ateles paniscus), in 1 sample of faeces from 7 adult squirrel monkeys (Saimiri boliviensis), in 1 sample from 2 females and 1 male tufted capuchin (Cebus apella), in 1 sample of feces from baboons (Papio hamadryas) and in the sample from 1 young male chimpanzee (Pan troglodytes).

Key words: Cryptosporidium, Protozoa, Survey, Animals from Zoo.

\section{INTRODUCCIÓN}

La cryptosporidiosis es una zoonosis de amplia distribución mundial, causada por diversas especies de Cryptosporidium, más frecuente en individuos jóvenes, que se caracteriza por ocasionar diarreas en seres humanos y otros mamíferos, aunque en algunas especies la infección cursa sin manifestaciones clínicas. Los trabajos publicados sobre cryptosporidiosis de animales en Argentina mencionan su detección en cerdos y bovinos. Esta parasitosis habría sido detectada por primera vez en el año 1983, en Argentina en animales domésticos ${ }^{3}$. Posteriormente, se confirma su participación como agente etiológico de la diarrea neonatal del ternero'. Actualmente son 13 las especies de Cryptosporidium que se consideran válidas, de ellas, 6 son zoonóticas $^{2}$ y ninguna de las 13 se puede distinguir por medio de estudios morfológicos o por el hospedador al cual parasitan.

\section{MATERIAL Y MÉTODOS}

Se procesaron 375 muestras de materia fecal (mf) de animales de la provincia de Buenos Aires, que fueron remitidas al servicio de diagnóstico de la Cátedra de Parasitología y Enfermedades Parasitarias, por las técnicas de Ritchie modificada y Ziehl-Neelsen modificada. Se obtuvieron datos de edad de los animales y de presencia o no de diarrea. En la mayoría de los casos se examinó una sola muestra por animal, en pocos casos se examinaron 2 muestras.

Todos los datos de las muestras de material fecal están en la Tabla 1.

\footnotetext{
* Cátedra de Parasitología y Laboratorio de Inmunoparasitología. Universidad de la Plata. Argentina.
} 
Técnica de Ritchie modificada: Las heces se mezclaron en proporción 1:10 con la solución salina de formol (formol: $50 \mathrm{mi}$, CI Na $5 \mathrm{~g}$, agua destilada $950 \mathrm{mI}$ ); la mezcla se filtró con malla metálica de $1 \mathrm{~mm}$ y se colocó en un tubo de centrífuga hasta las $3 / 4$ partes. Se agregaron 2 $\mathrm{ml}$ de éter sulfúrico, se centrifugó a $1.500 \mathrm{rpm}$ durante 3', se eliminó el sobrenadante, se tomaron gotas del sedimento con pipeta Pasteur y se observó al microscopio.

Técnica de Ziehl Neelsen modificada: Se dejó secar una gota de sedimento de mf en un portaobjetos, se cubrió con fucsina fenicada durante 5', se lavó con agua corriente y decoloró con alcohol clorhídrico al 3\%, se lavó con agua corriente y cubrió con azul de metileno al $1 \%$ durante 10', se lavó, secó y observó al microscopio ( 45 x y 100 x). Con esta coloración los ooquistes de Cryptosporidium $s p$ se tiñen de color rosado intenso o claro, con bordes definidos.

\section{RESULTADOS}

Se detectaron ooquistes en $19,46 \%$ del total de las muestras analizadas (73 de 375); en la Tabla 2 está la frecuencia de detección de ooquistes de Cryptosporidium sp según las especies.

Se hallaron ooquistes de Cryptosporidium $\mathrm{sp}$ en 2 perros de 1,5 meses, en 3 de 2,5 meses y en uno de 10 meses, una muestra no tuvo el dato de edad.

Se hallaron ooquistes en las dos muestras de $\mathrm{mf}$ de gato que se examinaron con intervalo de un mes.

En las muestras de las siguientes especies de monos, se detectó Cryptosporidium sp: la hembra carayá adulta; el mono araña adulto; la muestra colectiva de 7 saimiri adultos; la del chimpancé joven; la muestra colectiva de una madre hamadríade con crías y las de dos hembras y un macho caí.

Tabla 1. Datos de las muestras de materia fecal examinadas en este estudio

\begin{tabular}{|c|c|c|c|}
\hline Especie erro & n de Muestras & Edad & Observaciones \\
\hline Perro & 175 & 1 mes/10 años & \\
\hline Gato & 17 & sin datos & $\begin{array}{l}1 \text { animal con diarrea } \\
2 \text { muestras en } 1 \text { mes }\end{array}$ \\
\hline Cabritos & $\begin{array}{r}12 \\
5 \\
7+7\end{array}$ & $\begin{array}{l}\text { 20-30 días } \\
\text { 19-53 días } \\
\text { Hasta } 45 \text { días }\end{array}$ & $\begin{array}{l}\text { heces diarreicas } \\
\text { heces no diarreicas } \\
2 \text { muestras/animal } \\
\text { en } 22 \text { días }\end{array}$ \\
\hline Corderos & 22 & hasta 45 días & de 3 procedencias \\
\hline Terneros lecheros & 109 & 10-30 días & $\begin{array}{l}\text { heces diarreicas criados } \\
\text { en estaca, de } \\
8 \text { establecimientos }\end{array}$ \\
\hline Caballos & 2 & Adultos & heces diarreicas \\
\hline Cobayos & 5 & sin datos & \\
\hline Carayá (Aloualta caraya) & 2 & $\begin{array}{l}1 \text { adulto } \\
1 \text { joven }\end{array}$ & \\
\hline Araña (Ateles paniscus) & 1 & adulto & \\
\hline Saimiri (Saimiri boliviensis) & 2 & $\begin{array}{l}1 \text { muestra adultos } \\
1 \text { muestra adultos }\end{array}$ & $\begin{array}{l}12 \text { animales } \\
7 \text { animales }\end{array}$ \\
\hline Chimpancé (Pan troglodytes) & 2 & 1 joven 1 adulto & \\
\hline Diana (Cercopithecus diana) & 1 & adulto & \\
\hline Tití (Callithiyxjacchus) & 1 & adulto & \\
\hline Hamadríade (Papio hamadryas) & 2 & 1 muestra 1 muestra & $\begin{array}{l}\text { hembra y sus crías } \\
2 \text { adultos }\end{array}$ \\
\hline Caí (Cebus apella) & 3 & $\begin{array}{l}1 \text { muestra } 1 \text { muestra } \\
1 \text { muestra }\end{array}$ & $\begin{array}{l}2 \text { adultos } \\
4 \text { adultos } \\
3 \text { adultos }\end{array}$ \\
\hline
\end{tabular}


Cryptosporidium en animales domésticos y monos de un zoológico - L. Venturini et al.

Tabla 2. Cantidad de muestras en las cuales se hallaron ooquistes de Cryptosporidium sp. en sus heces (mf), según especies animales

\begin{tabular}{lccr}
\hline Mf de & con ooguistes & sin ooguistes & total \\
\hline perros & 7 & 168 & 175 \\
ovinos & 4 & 18 & 22 \\
equinos & 2 & 0 & 2 \\
cobayos & 2 & 3 & 5 \\
gatos & 2 & 15 & 17 \\
bovinos & 29 & 80 & 109 \\
caprinos & 21 & 10 & 31 \\
total & 73 & 301 & 375 \\
\hline
\end{tabular}

Tabla 3. Muestras de material fecal de terneros con ooquistes, de Cryptosporidium sp. por establecimiento

\begin{tabular}{lcrr}
\hline $\begin{array}{l}\text { Estable- } \\
\text { cimiento }\end{array}$ & $\begin{array}{c}\text { muestras } \\
\text { con ooquistes }\end{array}$ & total & $\%$ \\
\hline 1 & 2 & 30 & 6 \\
2 & 5 & 10 & 50 \\
3 & 5 & 7 & 71 \\
4 & 5 & 7 & 71 \\
5 & 2 & 9 & 22 \\
6 & 3 & 6 & 50 \\
7 & 7 & 20 & 35 \\
8 & 0 & 20 & 0 \\
total & 29 & 109 & 26,6 \\
\hline
\end{tabular}

Tabla 4. Muestras materia fecal de cabritos de 19 a 53 días de edad y presencia de ooquistes de Cryptosporidium sp

\begin{tabular}{lcccc}
\hline Cabritos & $\begin{array}{c}\text { Muestras con } \\
\text { ooguistes }\end{array}$ & $\begin{array}{c}\text { Muestras sin } \\
\text { ooguistes }\end{array}$ & Total & Edad días \\
\hline Heces diarreicas & 11 & 1 & 12 & $20-30$ \\
Heces no diarreicas & 3 & 2 & 5 & $19-53$ \\
\hline
\end{tabular}

Tabla 5. Muestras de materia fecal de 7 cabritos con intervalo de 22 días y presencia de ooquistes de Cryptosporidium sp

\begin{tabular}{lccc}
\hline Muestra & $\begin{array}{c}\text { Muestras con } \\
\text { ooquistes }\end{array}$ & $\begin{array}{c}\text { Muestras } \\
\text { sin ooquistes }\end{array}$ & Total \\
\hline $1^{\text {a: }: 31 \text { días }}$ & 6 & 1 & 7 \\
$2^{\text {a: }} 53$ días & 4 & 3 & 7 \\
\hline
\end{tabular}

En la Tabla 3 se hallan los datos sobre las muestras de $\mathrm{mf}$ de terneros con ooquistes, por establecimiento y en las Tablas 4 y 5 , los de caprinos.

\section{DISCUSIÓN}

Se comprobó la presencia de infecciones por Cryptosporidium sp en perros, ovinos, equinos, cobayos, gatos, bovinos, caprinos y en distintas especies de monos, en Argentina.

Los datos obtenidos sobre los signos clínicos y edad de los animales no fueron suficientes para evaluar la asociación de estos factores con la detección de ooquistes.

La primera mención de este parásito en animales domésticos en Argentina ${ }^{3}$. Fue hecha en el estudio histopatológico de un ternero enfermo de diarrea neonatal, mientras que la primera detección de ooquistes en exámenes de materia fecal, la habría realizado Bellinzoni RC y col, quienes confirmaron la asociación del parásito en la diarrea neonatal de los terneros ${ }^{1}$. Según este autor se detectaron $29,6 \%$ de animales infectados de 239 , valor cercano al obtenido en este trabajo que fue de $26,6 \%$ de 109 muestras de aspecto diarreico de terneros de tambo.

Las especies no fueron identificadas, pero a excepción de los cobayos, los otros hospedadores pueden estar infectados por especies zoonóticas ${ }^{2}$. En la mayoría de las infecciones de seres humanos se ha detectado $C$. hominis que también infecta a monos y ovinos, y $C$. parvum que se halla además en bovinos, ovinos, caprinos -y menos frecuentemente ciervos, ratones y cerdos. $C$. muris parasita principalmente a ratones y camellos y secundariamente a seres humanos; $C$. felis a gatos y secundariamente a humanos y bovinos; $C$. canis a perros y secundariamente a humanos. Los bovinos, ovinos y camellos pueden estar infectados por $C$. andersoni, que al igual que $C$. wrairi que infecta a los cobayos, no 
infecta a los seres humanos ${ }^{2}$. C. parvum, ha estado asociado con la presentación de brotes de la enfermedad en los seres humanos, ocasionados por la transmisión directa entre humanos, por animales o por agua mientras que las otras especies zoonóticas contribuyeron a la presentación de criptosporidiosis en los seres humanos en casos aislados ${ }^{2}$; por esta razón, para determinar el grado de riesgo que representan las especies de animales estudiadas de transmitir la infección a seres humanos, es necesario determinar cuales son las especies de Cryptosporidium que ocurren en los animales en Argentina, así como la presencia de otros factores de riesgo.

\section{RESUMEN}

El objetivo del trabajo fue detectar infecciones por Cryptosporidium sp en animales domésticos y en monos de un zoológico, en la provincia de Buenos Aires, Argentina.

Se procesaron 375 muestras de materia fecal de distintas especies mediante la técnica de sedimentación de Ritchie modificada (formol éter) para concentrar los ooquistes. El sedimento se tiñó mediante la técnica de Ziehl-Neelsen modificada.
Se detectaron ooquistes de Cryptosporidium sp en 7 de 175 muestras de materia fecal de perro, en 2 de 17 de gato, en 4 de 22 de ovinos, en 21 de 131 cabras, en 29 de 109 de terneros, en 2 de 2 de equinos y en 2 de 5 de cobayos (Cavia porcellus). Se examinaron 14 muestras de heces de monos, entre ellas, se detectaron ooquistes en la muestra de 1 hembra carayá (Alouatta caraya) adulta, en la de 1 mono araña (Ateles paniscus) macho adulto, en la muestra colectiva de 7 monos saimiri (Saimiri boliviensis) adultos, en la muestra de 2 hembras y 1 macho caí (Cebus apella), en la muestra colectiva de hamadríades (Papio hamadryas) y en la de 1 chimpancé joven (Pan troglodytes).

\section{REFERENCIAS}

1.- BELLINZONI R C, BLACKHALL J, TERZOLO H R, et al. Microbiology of diarrhoea in young beef and dairy calves in Argentina, Rey. Argent MicrobioL1990; 22: $130-6$.

2.- XIAO L, FAYER R, RYAN U, UPTON S J. Cryptosporidium Taxonomy: Recent Advances and Implications for Publio Health. Clinical Microbiology Reviews 2004; 17: 72-97.

3.- ODEÓN A C, MAGNASCO E J. Primera observación de Cryptosporidiosis en terneros enfermos de diarrea neonatal en la República Argentina. Gaceta Veterinaria 1983; Vol XLIVN 372: 670-3. 